\title{
Newly Found Literatures Owned by Ekai Kawaguchi Kept in the Rissho University Library
}

\author{
SHōJI Fumio
}

\section{Introduction}

Ekai Kawaguchi 河口慧海 (1866-1945) is well known as a man who brought Buddhist literatures from India, Tibet and Nepal to Japan. We know that the literatures brought by him are kept in the Toyo Bunko 東洋文庫, The University of Tokyo 東京大学, Taisho University 大正大学 and Tohoku University 東北大学. ${ }^{1)}$

In 2009, I investigated undisposed-of literatures kept in the Rissho Univeristy Library 立 正大学図書館. ${ }^{2)}$ I found literatures owned by Ekai Kawaguchi among them unintentionally. In reality, though the fact was written in the following book, little is known about the literatures, because they have never been studied so far.

In 1961, Akira Kawaguchi 河П正 (1918-1962) published a book written about his uncle Ekai Kawaguchi. ${ }^{3)}$ He had referred to the literatures kept in Rissho University in his book. According to the book, then, the literatures were kept in the house of Hanzui Kawaguchi 河口半瑞 (a younger brother of Ekai), in Tokyo and, after death of Ekai Kawaguchi, at "Research Institute for the Study of Mutual Influence of East and West 東西文化交 流研究所"4) in the Rissho University in 1952." Now they are kept in the Rissho University Library. ${ }^{6)}$

This paper is intended as an investigation of newly found literatures owned by Ekai Kawaguchi kept in the library. ${ }^{7)}$ The following is a list of the literatures and a transliterated text of Samvarodaya-tantra from Sanskrit manuscript.

\section{List of the Literatures}

\section{Sanskrit Manuscripts}

\section{Ganda-vyūha}

Paper, 401 folios (complete), 7 lines, $12.8 \times 45.0 \mathrm{~cm}$. Nepalese character. Wooden book 
cover. The first folio has a picture. [no date] Cf. Tamayose 1908a/b, Izumi 1910, Shöji 2010. It is the original text of The Gandavyuha sutra (part $I-I V$ ) edited by Suzuki \& Izumi 1934-36.

\section{Samvarodaya-tantra}

Paper, 1 folio (the fifth folio only), 6-7 lines, $7.5 \times 23.0 \mathrm{~cm}$. Devanāgarī. Cf. Matsunami's catalogue No.401-405 (Nos. 401-404 were brought by Ekai Kawaguchi). Cf. Tsuda 1974, pp.76-77. chapter 2-3.

\section{Pāli Manuscripts}

1. [unidentified]

Palm leaf, 78 folios, 4 lines, $3.6 \times 44.5 \mathrm{~cm}$, Sinhalese character. Wooden book cover.

2. [unidentified]

Palm leaf, 104 folios, 4 lines, $3.2 \times 44.0 \mathrm{~cm}$, Javanese character. Wooden book cover.

\section{3. [unidentified]}

Palm leaf, 116 folios, 4 lines, $3.7 \times 45.3 \mathrm{~cm}$, Javanese character. Wooden book cover.

\section{Tibetan literatures $^{8)}$}

\section{1. parts of sDe dge bka' 'gyur and bsTan 'gyur}

Pecha, 83 folios, 7 lines, $12.0 \times 61.0$ (print size: $7.4 \times 52.8) \mathrm{cm}$.

\section{2. bsTan bcos 'gyur ro cog gsung bar du bsgrubs pa'i dkar chag tshangs pa'i dbyangs}

Pecha, 135 folios (incomplete, $1-10$ folios are omited), $16.0 \times 65.5(8.5 \times 50.9) \mathrm{cm}$. Wooden book cover.

\section{3. 'Phags pa shes rab kyi pha rol tu phyin pa brgyad stong pa}

Pecha, 574 folios, $8.8 \times 30.8(4.5 \times 19.4) \mathrm{cm}$. Wooden book cover. = Walsh list No.64. Cf. Shōji 2011

\section{4. 'Phags pa dum bu zhe gnyis pa zhes bya ba'i mdo}

Pecha, 82 folios, $14.0 \times 56.0(11.0 \times 47.0) \mathrm{cm}$.

\section{5. [mDo mang] and dKar chag dgos 'dod kun 'byung}

Pecha, 716 folios, $16.7 \times 61.8(10.4 \times 50.2) \mathrm{cm}$. Wooden book cover. It consits of 95 volumes.

\section{6. 'Phags pa dzam bha la'i mtshan brgya rtsa brgyad pa}

Pecha, 3 folios, $16.7 \times 61.8(10.7 \times 51.8) \mathrm{cm} .=$ Toyo Bunko No.337B [?]

\section{Shes rab snying po'i ți ka}

Pecha, 7 folios, 6 lines, $9.7 \times 52.0(6.3 \times 47.0) \mathrm{cm} .=$ Toyo Bunko No. $340 \mathrm{~A}-\mathrm{E}$ 
8. dBu ma rtsa ba shes rab kyi ngag don bshad pa rin po che'i phreng ba

Pecha, 48 folios, $9.9 \times 59.2(6.1 \times 47.5) \mathrm{cm}$. $=$ Walsh list No.70.

9. [a-d] Byā ka ra na sum cu pa dang rtags kyi 'jug pa gnyis

Pecha, [a]-[b] 4 folios, 6 lines, $8.4 \times 53.2(5.7 \times 46.8) \mathrm{cm}$. = Toyo Bunko No.390B $\doteqdot \mathrm{C}$.

[c] 4 folios, 6 lines, $10.3 \times 56.0(7.0 \times 51.2) \mathrm{cm}$. = Walsh list No.25-1.

10. [a-b] Bod kyi brda sprod pa'i gzhung sum cu pa dang rtags kyi 'jug pa nyung ngu'i tshig gis go sla bar bkral ba legs bshad snang ba dam pa

Pecha, [a] 49 folios, 6 lines, $8.7 \times 45.0(5.8 \times 37) \mathrm{cm}$. [b] 49 folios, 6 lines, $10.7 \times$ $56.5(7.2 \times 51.3) \mathrm{cm} .=$ Walsh list No.26, Toyo Bunko No.392A-C.

11. [a-b] Yul gangs can gyi skad kyis brda sprod pa'i bstan bcos sum cu pa dang rtags kyi 'jug pa'i rnam bshad mkhas mchog si tu'i zhal lung

Pecha, $[\mathrm{a}]=[\mathrm{b}] 26$ folios, 6 lines, $9.0 \times 54.5(7.0 \times 50.4) \mathrm{cm} .=$ Walsh list No.25-2, Toyo Bunko No.393A = 395A, Tohoku No. 6388 .

12. Lung du ston pa sum cu pa dang rtags kyi 'jug pa'i rnam 'grel legs bshad snang byed nor bu

Pecha, 64 folios, 6 lines, $9.0 \times 54.0(5.8 \times 46.7) \mathrm{cm} .=$ Toyo Bunko No.393B $=395 \mathrm{~B}$.

13. Sum rtags kyi dper brjod mkhas pa'i yid 'phrog

Pecha, 4 folios, 6 lines, $8.3 \times 53.0(5.8 \times 46.8) \mathrm{cm} .=$ Toyo Bunko No.396B.

14. Sum rtags kyi rnam bshad nor bu ke ta ka'i do shal du 'bod pa'i dgag lan tshangs pa'i thig gi spun zla

Pecha, 8 folios, 6 lines, $11 \times 57.3(6.8 \times 52.0) \mathrm{cm}$. = Toyo Bunko No.397.

15. sGra'i bstan bcos sum cu pa'i tshig don gsal ba'i me long ${ }^{9)}$

Pecha, 19 folios, 6 lines, $8.3 \times 53.3(5.9 \times 47.2) \mathrm{cm}$. $=$ Toyo Bunko No.400A = B. Cf. Shōji 2010, 36-38.

16. Sum cu pa dang rtags kyi 'jug pa'i go don rtso ma rgyugs su byed pa'i dper brjod la phran bu'i 'grel bshad dang bcas pa legs bshad rin chen do shal

Pecha, 32 folios, $9.8 \times 56.9(7.8 \times 49.2) \mathrm{cm}$.

17. rTags kyi 'jug pa'i snying po'i don mdo tsam brjod pa dka' gnad gsal ba'i me long

Pecha, 5 folios, 6 lines, $10.6 \times 57.0(7.2 \times 49.4) \mathrm{cm}$.

18. [a]-[c] sGra'i bstan bcos rtags kyi 'jug pa'i tshig don gsal ba'i me long

Pecha, $[\mathrm{a}]=[\mathrm{b}]=[\mathrm{c}] 16$ folios, 6 lines, $8.2 \times 53.5(5.8 \times 47.3) \mathrm{cm} .=$ Toyo Bunko 401 


\section{Bod kyi brda'i bye brag gsal bar byed pa ngag gi sgron ma}

Pecha, 24 folios, 6 lines, $8.7 \times 53.4(6.8 \times 49.2) \mathrm{cm}$. = Toyo Bunko No.406, Otani No.11869, Tohoku No.7065.

20. Bod kyi brda'i bye brag gsal bar byed pa'i bstan bcos tshig le'ur byas pa mkhas pa'i ngag gi sgron ma

A folding book, $13.5 \times 30.5 \mathrm{~cm}$.

21. Bod kyi skad las gsar rnying gi brda'i khyad par ston pa legs par bshad pa li shi'i gur khang

Pecha, 15 folios, 6 lines, $9.8 \times 54.6(6.3 \times 47.3) \mathrm{cm} .=$ Tohoku No.7064.

22. (1) sDeb sbyor rin chen 'byung gnas kyi bsdus 'grel kun phan snang ba

Pecha, 51 folios, 6 lines, $8.7 \times 44.4(5.7 \times 37.4) \mathrm{cm}$.

\section{Transliteration of Samvarodaya-tantra}

Cf. Tsuda 1974, pp.76-77. (Eng. tr. pp.242-244)

(r.1) karmabījavasāt prāptavāyavo parivarttya ca || dharmodayā yonidvārāṇām abhimukham bhavati niścitam || dakṣiṇakukșim samāśritya utkutkasthitam abhisanmukham \| vāmakukșim samāśritya prajñā udaramukhī bhavet || bījādhānakrame kāle muhūrtta-lakșayet sudhīh || dakșiṇe vahati yo vāyuh praruṣo bavati sarvadā vāme vahati yo mā $\langle$ ru $\rangle \mathbf{t}$ strīnām bhavati niścitam \|| ubhayor madhyagatam bījạ̣ na puṃsakaṃ sadā bhavet || apu dhātuh paitṛko jñeyā tejo-dhātuś ca mātṛkā || tvan mānsaś ca raktaś ca mātrjā iti kathyate || snnāyu majjañ ca śukram ca pitṛā iti kathyate \| evam șaṭauśikam piṇḍạ vajrasatvavaco yathā || rūpa-veda(v.1) nā samjjñā saṃskāra vijñānam e 〈va $\rangle$ ca || pạ̣ca-buddha-svabhāvaṃ tu skaṃdhôtpatti-viniścitāh \| janmôtpatti-kramaṃ jñātam saṃmyaksaṃbuddhatvam āptuyāt $\|$ etat skaṃdha-parijñānam kathitạ̣ tat vavādinā || || iti utpatti-nirdeśah pațalah dvitìyah \| \| athāsampravakṣāmi utpanna-krama-bhāvanām || yena vijñātramātreṇa āśu siddhim avāpnuyāt || kāyamaṇ̣alam āśritya dharmasaṃbhogavigrahaị || dehamaṃ̣alam ity uktam saṃbodhikrama-sādhanaṃ || utpatti-mṛdu-madhye yogī dhyāyān maṃ̣ala-bhāvaṇām || adhimātre jhațitākāram mạ̣ḍalạ̣ citta-mātratā || jhaṭitākārayogena utpaṃna-krama-bhāvanā || traidhātukam ayaṃ kūṭam prāṇino māṇụaleyakā || tanmadhye jhaṭitākārạ̣ yogī syāt maṇḍalādhipaḥ || oṃ āḥ hūṃ iti mantreṇa kāyavākcitta-maṇ̣alaṃ || svargasarvaṃ ca pātāla 


\section{Notes}

1) Cf. Akira Kawaguchi 1961, pp.168-174; 2000, pp.216-227.

2 ) i.e. Rissho University for Center for Information and Media Osaki Library.

3) Akira Kawaguchi 1961.

4) This institute published the journal, Bunka-koryu 文化交流, from 1952 to 1953. People who contributed to this institute were as follows: Hiroto Saigusa 三枝博音 (professor, R[issho]. Univeristy), J.R. Brinkley (professor, R. Univ.), Takashi Sasaki 佐々木峻, Tanzan Ishibashi 石橋湛山 (the president of R. Univ., from 1952 to 1968), and so on (Cf. Bunka-koryu, 1 (1), 1952, p.31).

5 ) Some books of the Kawaguchi collection were given to "Research Institute for the Study of Mutual Influence of East and West" that was established in the Rissho University (the abstract from Akira Kawaguchi 1961, pp.173-174; 2000,pp.226-227). Tanzan Ishibashi 石橋湛山 (1884-1973), who was a member of the institute, referred to the Kawaguchi collection 河口恵 [sic.]海文庫 in his diary (Cf. Ishibashi 2001a, p.439, p441, p.468, and 2001b, p.501).

6 ) Cf. Shōji 2010, pp.36-38.

7 ) This document has a short note in the title page by Śrī Sārat Chandra in Tibetan language as follows:

rdo rje gling lha sa bi lla la bzhug pa'i skabs su phun sum tshogs pa shar phyogs bhang ga la'i yul gyi pan di ta shrī sha ra ta tsan dras ja pan yul gyi mkhan chen bla ma shes rab rgya mtsho ming can e ka'i ka ba gu ci la phul lo/ 17th may 1911. (Its picture has published in Shōji 2010, p.37)

Translation: "When Kawaguchi stayed at Lhasa Villa in Darjeeling, [this document was] gifted to Shes rab rgya mtsho (Ekai, Japanese monk) by Pandit Śrī Sārat Chandra (West Bengal) 17th may 1911." 8 ) Cf. Walsh 1904 (= Walsh list) and Toyo Bunko 2002.

9 ) I am now writing a book about this study Descriptive catalogue of Ekai Kawaguchi collection kept in the Rissho University Library 河口慧海文庫解題目録 that will be published in 2012.

\section{References}

Ishibashi, T[anzan]. 石橋湛山

2001a/b The diary of Tanzan Ishibashi, vol. 1 and 2 石橋湛山日記 上/下. Tokyo: Misuzushobo. Izumi, H[okei]. 泉芳璟

1910 “On Sanskrit languages 梵語はなし草.” Mujintō 無盡燈 15 (6), pp.46-49.

1928 Ganda Vyūha, [s.1.] 1488 pages.

Kawaguchi, A[kira]. 河口正

1961 Ekai Kawaguchi 河口慧海. Tokyo: Shunjūsha (reprint, 2000).

Shōji, F[umio]. 庄司史生

2010 “Travelling books 旅する本." In An illustrated book of exhibition in the Rissho University Library 立正大学大崎図書館今昔蔵書選. Tokyo: Rissho University Library, pp.36-38.

2011 "On the 'Phags pa shes rab kyi pha rol tu phyin pa brgyad stong pa brought to Japan 
(164) Newly Found Literatures Owned by Ekai Kawaguchi Kept in the Rissho University Library (F. SHōJI)

by Ekai Kawaguchi preserved in Library of Rissho University 立正大学図書館所蔵・ 河口慧海将来チベット語訳『八千頌般若経』.”Journal of Buddhist Studies: Bukkyōgaku Ronshū 仏教学論集 28, pp.1-16.

Suzuki, D[aisetsu]. T[eitaro]. \& Izumi, H[okei].

1934-36 The Gandavyuha sutra (part I-IV). Kyoto: Sanskrit Buddhist Texts Pub. Society (New rev. ed., 1949).

Tamayose, H[ōun]. 玉代勢法雲

1908a/b “A study of Sanskrit texts of the Kegon Sutra 梵本華綮経の研究.” Mujintō 無盡燈 $13(5), \mathrm{pp} .2-24 / 13(8), \mathrm{pp} .11-27$.

Toyo Bunko 東洋文庫

2002 "Description of Kawaguchi Collection preserved in the Toyo Bunko 河口請来蔵外文 献解説.” (http://61.197.194.9/Database/Kawaguchi_collection.pdf)

Tsuda, S[hiníchi].

1974 The Samvarodaya-tantra: selected chapters. Tokyo: Hokuseido.

Walsh, E[rnst]. H [erbert]. C [ooper].

1904 "A list of Tibetan Books brought from Lhasa by Japanese Monk, Mr. Ekai Kawa

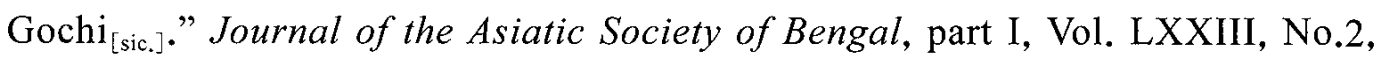
pp.118-177.

〈Acknowledgements〉 I would like to thank Rissho University Library for the use of the literatures.

〈Key words〉 Ekai Kawaguchi, Ganda-vyūha, Samvarodaya-tantra

(Part-time Lecturer, Rissho University) 